\title{
The first case of drug-induced pseudoscleroderma and eczema craquelé related to nab-paclitaxel pancreatic adenocarcinoma treatment
}

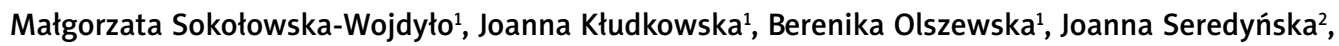 \\ Wojciech Biernat ${ }^{3}$, Izabela Błażewicz ${ }^{1}$, Alicja Rustowska-Rogowska ${ }^{1}$, Roman J. Nowicki ${ }^{1}$
}

${ }^{1}$ Department of Dermatology, Venereology and Allergology, Medical University of Gdansk, Gdansk, Poland

${ }^{2}$ Regional Oncology Center, Gdansk, Poland

${ }^{3}$ Department of Pathology, Medical University of Gdansk, Gdansk, Poland

Adv Dermatol Allergol 2018; XXXV (1): 106-108

DOI: https://doi.org/10.5114/pdia.2017.67054

We report a case of drug-induced pseudoscleroderma and eczema craquelé after treatment with nab-paclitaxel for metastatic pancreatic ductal adenocarcinoma. This case report is, according to our knowledge, the first to link solvent-free paclitaxel with pseudoscleroderma and eczema craquelé.

A 68-year-old patient with pancreatic ductal adenocarcinoma (the patient underwent total pancreatectomy 42 months ago, followed by chemotherapy with gemcitabine for 6 months) was admitted to the Department of Dermatology, Venereology and Allergology for further diagnosis of skin lesions. The skin lesions clinically appeared as extensive sclerosis distributed on the feet, especially the dorsal part (Figure 1 A), legs (Figure 1 B), knees and inner thigh area (Figure $1 \mathrm{C}$ ). They had progressed over the course of 10 months. Keratinization and eczema craquelélike lesions (Figure 1 B) were observed locally. Slight skin burning sensation, skin tension, pain and limitation of the ankle and metatarsophalangeal joint movement accompanied skin lesions. Moreover, the left hand remained swollen for several months. Physical examination revealed hyperpigmentation of the skin all over the body and cachexia. The patient was diagnosed with diabetes 10 years ago. The patient remains under the care of the Regional Oncology Center, where she is undergoing treatment with nab-paclitaxel and gemcitabine, due to pancreatic cancer with lymph node metastasis confirmed by fine-needle aspiration biopsy and imaging studies.

The first skin lesions in the form of redness and swelling had developed initially over the lower extremities and then as generalized hyperpigmentation disseminated across the body, which occurred 2 months after the first administration of the current nab-paclitaxel and gemcitabine chemotherapy (treatment had been continued for 12 months; 36 rounds of the drug had already been given). Over time the skin of the lower extremities became hard. Induration of the skin proceeded from the feet to the thighs. Raynaud's phenomenon did not occur. Doppler ultrasound of lower limb veins and arteries was within normal limits.

Basic laboratory and microbiological examinations were performed in the Dermatology Clinic. Laboratory investigations revealed microcytic anemia (a hemoglobin $(\mathrm{Hb})$ level of $9.7 \mathrm{~g} / \mathrm{dl}$; a hematocrit level of 31.6\%; MCV 75.6 fl); leukopenia (total leukocyte count of $3.69 \times 10^{-9} /$ l), hypoalbuminemia (serum albumin count of $29 \mathrm{~g} / \mathrm{l}$ ). Laboratory examinations included an antinuclear antibody test with positive titer ANA-Hep2: $1: 1280$ and fine-grained nucleolar pattern; the extended immunoblot scleroderma antibody profile was negative. In order to exclude pretibial myxedema, examination of thyroid hormone levels was performed - the results were normal. Lesional punch biopsy of affected skin documented thickened and strongly sclerotic skin with corneum deposits and without clear inflammatory infiltrates. The image corresponded to the diagnosis of localized scleroderma/ pseudomorphea (Figure 1 D).

The patient was treated with intravenous pentoxifylline and topical 20\% urea ointment, steroid ointment (clobetasol propionate), and moisturizing creams, which eventually resulted in resolution of sclerotic scale, gradual softening of the skin and restoration of the range of motion of the ankle and metatarsophalangeal joints. Due to tense bullae that emerged on the skin of the ankles and lower extremities, a punch biopsy was taken again for histopathological examination. Thickened and acanthotic epidermis with superficial peeling of protein masses and swollen skin warts with a few lymphocytes surrounding the vessels were observed. The whole image

Address for correspondence: Berenika Olszewska MD, Department of Dermatology, Venereology and Allergology, Medical University of Gdansk, 7 Dębinki St, 80-952 Gdansk, Poland, phone: +48664169 111, e-mail: berenika.olszewska@o2.pl Received: 15.09.2016, accepted: 21.12.2016. 

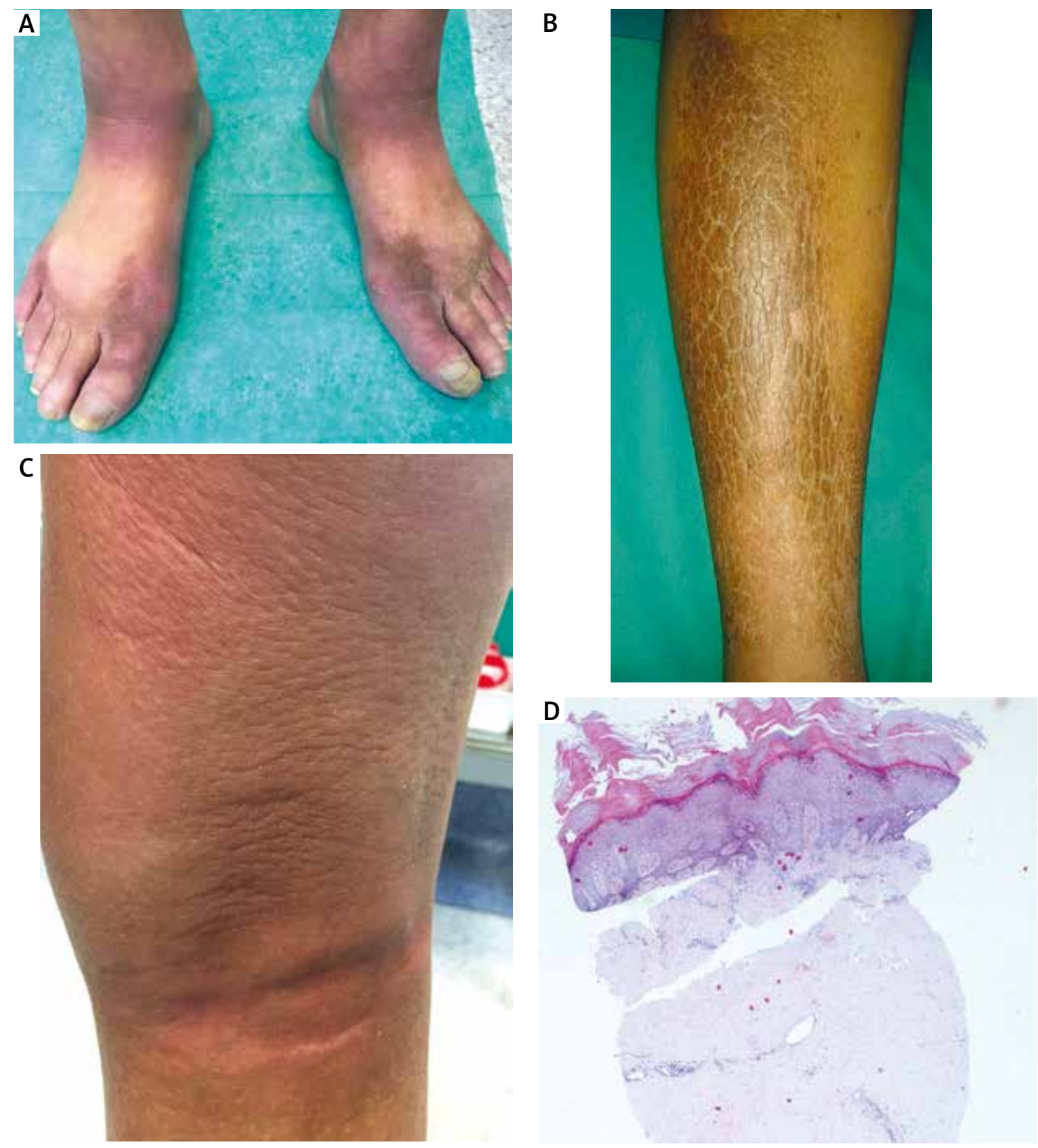

Figure 1. A - Sclerosis distributed on the dorsal part of feet and ankle had progressed over the course of 10 months after starting gemcitabine plus nab-paclitaxel chemotherapy. B - Lesions with so-called paving-stone or bed of a dry lake appearance on the lower extremities characteristic for eczema craquelé. C - Induration of the skin with orange peel appearance. Pretibial myxedema was proved negative. D - The biopsy shows hyperplastic epidermis covering densely sclerotic dermis $(H+E$, original magnification $40 x)$

led to the diagnosis of eczema craquelé. The patient was referred to the Outpatient Dermatology Clinic to continue treatment. Oral pentoxifylline at a dose of $400 \mathrm{mg}$ daily and topical therapy was continued. Six weeks after hospital discharge, the patient developed persistent blisters and erosions, as well as the intensification of skin scle- rosis. The oncologist referred the patient to a consultant dermatologist, who recommended topical glucocorticoid therapy together with reduction of the INN-paclitaxel dose (from $188 \mathrm{mg}$ to $140 \mathrm{mg}$ ).

According to published reports, several cases of scleroderma/pseudoscleroderma have been induced by taxane 
therapy while some cases have demonstrated the presence of ANA-Hep2 antibodies [1-3]. Only one case report describes involvement of gemcitabine [4]. According to some previously described cases, skin lesions initially presented as swelling, redness and accompanying hyperpigmentation, just like in our patient. Moreover, lesions with a paving-stone appearance were observed [5]. Development of scleroderma lesions during taxane therapy is dose-dependent and usually the lesions are not generalized [6].

There are also reports of scleroderma as a paraneoplastic syndrome [7]. However in our case the correlation between development of skin lesions after chemotherapy and progression of skin changes during treatment indicates an association with drugs. Moreover, improvement of the skin after 3 rounds of INN-paclitaxel at a reduced dose confirms it. On the other hand, the advancement of cancer and progression of skin lesions might suggest simulaneous progression of adenocarcinoma (the patient is waiting for the results of abdomen and chest computed tomography).

On the other hand, symptoms such as eczema craquelé, also referred to as asteatotic eczema, on the front surface of the lower extremities are typical for people over 60 years old. It is mostly a result of skin dryness, loss of the sebaceous glands and activity of sweat glands. Moreover, it is enhanced by external factors, such as high temperature and low humidity, the use of degreasing soaps and detergents, or long lasting baths in water at high temperature. Transepidermal water loss in lesional skin is 75-fold higher than in healthy skin. The skin presents lipid disorders (such as lower content of fatty acids: linoleic and linolenic acids), which disrupts the integrity of the dermo-epidermal barrier. Classic eczema craquelé is easy to treat, mainly by limiting harmful external factors and maintaining proper skin care (oiling, moisturizing, lower bath temperature, etc.). In some cases eczema may also be associated with poor nutrition - which in this case is associated with the underlying disease. Also, in some cases eczema craquelé has been reported to have been triggered by cancers: lymphomas, breast, lung, stomach, colon cancer or glucagonomas. Our patient's lesions with paving-stone or bed of a dry lake appearance may also be a paraneoplastic symptom of adenocarcinoma, or may be associated with cachexia.

Paradoxically, the mechanism of the anti-tumor activity of the drug may also be responsible for the development of scleroderma. Taxane-induced increase of proinflammatory cytokines such as tumor necrosis factor- $\alpha$ (TNF- $\alpha)$, IL-2, IL-6, and interferon- $\gamma($ INF- $\gamma$ ) was also observed in scleroderma [2]. Research on versican, chondroitin sulfate proteoglycan, might be the answer to these questions. Increased levels of versican and glycosaminoglycans in skin cells of patients with taxaneinduced lesions have been observed [6]. Similarly, high levels of glycosaminoglycans and versican in cells responsible for the excessive fibrosis are present in scleroderma.
Versican's main role is to stabilize the fibers of the skin by binding to extracellular proteins. Its can lead to excessive deposition of collagen in scleroderma. Histopathological examination of our patient's skin biopsy also revealed the concentration of protein masses. We still do not know why in taxane-induced scleroderma we do not observe either severe fibrosis in microvessel endothelial cells of skin or organ damage [5].

Our patient is being treated with gemcitabine plus nab-paclitaxel chemotherapy; 12 cycles have already been administered (on days 1, 8, and 15 of a 28-day cycle), which is currently the first-line treatment in patients with unresectable and metastatic pancreatic cancer. The combination of gemcitabine plus nab-paclitaxel chemotherapy has shown clinically relevant survival benefit over the standard gemcitabine monotherapy [8]. Due to the fact that chemotherapy of the underlying disease needs to be continued and therefore we cannot eliminate the cause of pseudoscleroderma, dermatological treatment can only reduce the symptoms to improve the quality of life of patients suffering from cancer.

\section{Acknowledgments}

This work was supported by funds from grant 020066/07 that has been received from the Polish Ministry of Science and Higher Education.

\section{Conflict of interest}

The authors declare no conflict of interest.

\section{References}

1. Pedersen JV, Jensen S, Krarup-Hansen A, et al. Scleroderma induced by paclitaxel. Acta Oncol 2010; 49: 866-8.

2. Bouchard SM, Mohr MR, Pariser RJ. Taxane-induced morphea in a patient with CREST syndrome. Dermatol Reports 2010; 2: 9.

3. Torregrosa JL, Fernandez M, Garcias J, et al. Morphea type plaques induced by paclitaxel. Med Clin (Barc) 2014; 142: 424-5.

4. Bessis D, Guillot B, Legouffe E, et al. Gemcitabine-associated scleroderma-like changes of the lower extremities. J Am Acad Dermatol 2004; 51: 73-6.

5. Takahashi T, Asano Y, Ichimura Y, et al. A case of taxaneinduced scleroderma: a different expression profile of Fli 1 proteins in dermal fibroblasts and microvascular endothelial cells compared with systemic sclerosis. Br J Dermatol 2011; 164: 1393-5.

6. Okada K, Endo Y, Miyachi Y, et al. Glycosaminoglycan and versican deposits in taxane-induced sclerosis. $\mathrm{Br}$ I Dermatol 2015; 173: 1054-8.

7. Jedlickova H, Durcanska V, Vasku V. Paraneoplastic scleroderma: are there any clues? Acta Dermatovenereol Croat 2016; 24: 78-80.

8. Goldstein D, El-Maraghi RH, Hammel P, et al. Nab-paclitaxel plus gemcitabine for metastatic pancreatic cancer: long-term survival from a phase III trial. J Natl Cancer Inst 2015; 107 : pii: dju413. 